\title{
Acoustic and semantic similarity effects on repetition avoidance in produced sequences
}

\author{
S. WIEGERSMA \\ University of Utrecht, Utrecht, The Netherlands
}

\begin{abstract}
The question of whether repetition avoidance in sequential response production depends on the phonetic or the semantic encoding of previous responses was investigated by varying the acoustic and semantic similarity among the response alternatives. The results indicated that acoustic similarity affected repetition avoidance with six alternative words and a production rate of one per second, but not with four alternative letters and a rate of one per 2 sec. Semantic similarity between words was also studied, and was not seen to affect repetition avoidance. Results were explained by meane of a model in which comparisons between a memory set of admissible responses and a memory set of recent responses are made at a phonetic level of response representation.
\end{abstract}

In sequential response production, the subject produces a sequence of responses selected from a set of designated responses. Repetition avoidance is the main characteristic of sequences produced in this way. Responses produced up to six trials earlier are avoided to some degree (Wagenaar, 1970; Wiegersma, 1982b). It seems likely that the hypothetical repetition-avoidance mechanism retains information about the previous responses and compares this with the memory set of admissible responses. The admissible responses are memorized in advance, such that they remain available during task performance. In other words, they have to be memorized for long-term, rather than for short-term, retention. However, it is assumed that the admissible responses are continuously available during task performance and need not be retrieved from long-term memory every time they are needed for comparison with the previous responses. The analysis of the response-production task used in this study is to a considerable degree similar to the analysis of the serial organization of words in language production. In the production of language, for example, in speech, words have to be selected from a set of possibilities that have been activated by the intentions of the speaker. Once these words have been retrieved from longterm memory, they (or a subset of them) have to be put in a serial order that expresses the thoughts of the speaker. For this to be done, the words not yet selected have to be compared with the ones already selected. It is in this hypothetical comparison of two memory sets of words that there is a functional correspondence with the sequential-response-production task. Of course, such lexical processes are not identical in sequential response productions of the type used in this study and in language production. Rather, it is assumed that the basic pro-

The author's mailing address is: Psychologisch Laboratorium, University of Utrecht, Varkenmarkt 2 Utrecht 2501, The Netherlands.

-Steven W. Keele served as Action Editor for this manuscript. cesses of retention, search, and comparison are used in both tasks, although the organization of these processes may be quite different for each of these activities. For example, constraints of syntaxis and meaning will have special effects on the serialization of words in the production of language. The sequential-response-production task presents the opportunity to study lexical selection processes free from such influences. However, this means that this task can only be used to study the general aspects of lexical selection processes, such as the type of memory representation involved. It seems therefore that the investigation of repetition avoidance in sequential word production can contribute to our knowledge of lexical processes in production. Another reason for studying repetition avoidance is that it shows up in a number of tasks other than response randomization, such as in absolute judgments (Wagner \& Baird, 1981) and in parapsychological studies (Solomon, 1949). In choice reaction time, a memory search process for finding nonrepetitions was proposed by Keele (1969). Thus, studying the mechanisms of repetition avoidance may contribute in two ways to our knowledge. First, it may contribute to our knowledge of the basic processes of planning response sequences. Second, it may contribute to models of how repetition avoidance comes about in the tasks mentioned above.

This study is directed at the nature of the memory representation used in repetition avoidance. Does repeti. tion avoidance occur at a semantic or at a phonetic level of representation? Such a question is related to the question of whether repetition avoidance is mediated by short-tcrm or long-term retention of previous responses. It has been suggested (Baddeley, 1976) that short-term retention typically uses phonetic memory codes, whereas long-term retention uses semantic codes. That is, shortterm retention retains the sounds of words, and longterm retention retains their meaning. This is in line with findings that long-term retention is negatively affected 
by semantic similarity of material, and short-term retention by acoustic similarity. However, occasional exceptions to this simple picture have been found, and it is not yet completely clear what the conditions are in which the one or the other type of coding will be used (Baddeley, 1976, pp. 114-120). In the production task used in this study, an indirect measure of the operation of memory is applied: The subject produces responses which have not recently been produced. But, for the subject to do so, the recent responses somehow have to be used for comparison. It is assumed that the subject does this by searching through recent responses. Thus, this task gives an opportunity to study the effects of acoustic and semantic similarity on search through memory, rather than on retrieval from memory, as in more direct studies of similarity effects on memory. Of course, what both tasks, sequential production and recall, have in common is the retention of previous responses. An acoustic-similarity effect in repetition avoidance can therefore be explained as an effect on the phonetic representation of previous responses, and not as a retrieval effect.

Two pilot studies were performed. In the first, subjects produced sequences of 120 responses that they selected at a rate of 1 every $2 \mathrm{sec}$ from one of five different sets of 4 responses. In order to make the conditions less dependent on accidental peculiarities, two equivalent sets of words were used in each condition. Each set was assigned to one-half (7) of the 14 subjects in each condition. The five types of words were: semantically dissimilar Dutch content words [equivalent sets: GIF (POISON), RAM (BUCK), SOK (SOCK), PUL (JUG), and KIN (CHIN), RAT (RAT), MOF (MUFF), BES (BERRY)] ; nonwords obtained by reversing the letter order of the dissimilar words (FIG, MAR, KOS, LUP, and NIK, TAR, FOM, SEB); acoustically different letters $(\mathrm{B}, \mathrm{Q}, \mathrm{N}, \mathrm{H}$, and $\mathrm{D}, \mathrm{X}, \mathrm{H}, \mathrm{Z})$; alliterated letters (L, R, S, M, and F, N, L, $\mathrm{R}^{1}$ ); and rhyming letters (B, G, $J, T$, and $G, D, P, V^{2}$ ). In this pilot study, neither semantic nor acoustic similarity affected the degree of repetition avoidance. It was hypothesized that the reason for the absence of the predicted effect of acoustic similarity on repetition avoidance might be that the memory load imposed by the task was too small. Perhaps a similarity effect would show up only if the processing and storage capabilities of the subjects were sufficiently loaded. Therefore, the experiment was repeated with a larger set of response alternatives (6) and an increased production rate.

As did the first pilot study, the second used semantically dissimilar Dutch content words [GIF (POISON), RAM (BUCK), SOK (SOCK), BED (BED), PIJL (ARROW), TUIN (GARDEN), and KIN (CHIN), MAF (SLEEPY), ROS (STEED), BEL (BELL), DIJK (DIKE), LUID (LOUD)] and nonwords obtained by inversion of the phoneme order of the words (FIG, MAR, KOS, DEB, LIJP, NUIT, and NIK, FAM, SOR, LEB, KIJD, DUIL). The acoustic conditions now used content words in- stead of letters: different words [BEK (MOUTH), MAF (SLEEPY), DOS (DRESS), ROET (SOOT), PIL (PILL), GIER (VULTURE), and FEL (FIERCE), DAM (DAM), ZOT (SILLY), ROEK (ROOK), MIN (MEAN), WIER (SEAWEED)], alliterated words [DOK (DOCK), DOL (MAD), DOS (DRESS), DOR (DRY), DOG (MASTIFF), DOP (SHELL), and MAK (TAME), MAF (SILLY), MAL (FOOLISH), MAN (MAN), MAP (FILE), MAT (MAT)], and rhyming words [HOK (SHED), SOK (SOCK), GOK (CHANCE), MOK (MUG), DOK (DOCK), ROK (SKIRT), and KAM (COMB), RAM (BUCK), DAM (DAM), LAM (LAMB), HAM (HAM), TAM (TAME)] . In this second pilot study, the production rate was one per second. The results again showed no significant effect of the semantic conditions $(F<1)$. However, repetition avoidance was now stronger in the acoustically different condition than in the acoustically similar conditions (alliteration and rhyme), as was shown by the significant a priori contrast $1,--.5,-.5[\mathrm{t}(31)=2.46, \mathrm{p}<.01]$.

Thus, this second pilot study showed the predicted effect of acoustic similarity and, as was expected, no difference between the semantic conditions. How. ever, a final experiment was added for two reasons. First, the difference between the semantic conditions (words vs. nonwords) was not on the dimension of meaningfulness of the items alone. Another difference was the frequency of use of words and nonwords in language. Sumby (1963) found that frequency may have a beneficial effect in short-term-memory tasks, at least in the primacy part of a serial position recall curve. It is not clear whether such a result can be generalized to the continuous sequential-response-production task. But the possibility cannot be excluded that frequency had a comparable effect in our task or that it might have had a quite different effect that could not be predicted on the basis of the present knowledge about the role of memory in repetition avoidance. Therefore, the semantic factor was varied in a different way in the experiment presented below: The word frequencies of the conditions were matched pairwise. Second, it was felt that the finding of a difference between acoustically different and similar items needed further support, since such a difference was found in the second, but not in the first, pilot study.

\section{METHOD}

The subjects had to produce series of 120 words selected from a set of 6 admissible words (Table 1). All words were onesyllable Dutch words.

The semantically related words all referred to the size of things: Set (a)-big; Set (b)-small. The semantically dissimilar words were selected in such a way that they not only were dissimilar, but also were not related by associations of contrast or by belonging to a common cognitive category. Moreover, their frequency in language was carefully matched to the frequency of the semantically similar words by using a frequency count of spoken Dutch words (Uit den Boogaart, 1975). The words in the three acoustic conditions were matched in the same way. 
Table 1

The Response Sets Used in Five Conditions

(English Translations Are in Parentheses)

\begin{tabular}{cl}
\hline $\begin{array}{c}\text { Conditions } \\
\text { Similar }\end{array}$ & (a) $\begin{array}{l}\text { groot-hoog-dik-breed-wijd-fors } \\
\text { (big-high-thick-broad-wide-robust) }\end{array}$ \\
Dissimilar & (b) $\begin{array}{l}\text { klein-laag-nauw-dun-smal-plat } \\
\text { (small-low-narrow-thin-narrow-flat) } \\
\text { goed-hoog-blij-koud-droog-leeg } \\
\text { (good-high-glad-cold-dry-empty) } \\
\text { klein-zwart-duur-trots-leeg-rond } \\
\text { (small-black-expensive-proud-empty- } \\
\text { round) }\end{array}$ \\
Acoustic & (a) $\begin{array}{l}\text { zuur-lok-roos-kam-stoep-echt } \\
\text { (sour-curl-rose-comb-sidewalk-real) }\end{array}$ \\
Different & (b) $\begin{array}{l}\text { kooi-buis-ton-dak-guur-lust } \\
\text { (cage-tube-cask-roof-raw-delight) } \\
\text { dooi-doof-dook-doop-doos-dood } \\
\text { (thaw-deaf-dived-baptism-box-dead) }\end{array}$ \\
Rhyme & (b) $\begin{array}{l}\text { leek-leed-leen-leeg-leef-lees } \\
\text { (layman-grief-borrow-empty-live-read) } \\
\text { dooi-gooi-fooi-hooi-prooi-mooi } \\
\text { (thaw-throw-tip-hay-prey-handsome) }\end{array}$ \\
& (b) $\begin{array}{l}\text { was-ras-jas-gas-bas-das } \\
\text { (wax-race-coat-gas-bass-tie) }\end{array}$
\end{tabular}

Note-(a) and (b) designate equivalent response sets.

Fourteen undergraduate psychology students were assigned to each of the semantic conditions; eight were assigned to each of the acoustic conditions. One subject of the alliteration condition was canceled because of a deviant interpretation of the instruction.

The subjects were instructed to use "no definite systems" in producing the sequence, and to produce their responses in pace with a regular light signal that came on once every second. The six response alternatives were presented one below the other on a card in $7-\mathrm{mm}$ black capitals. They had to be memorized before the subjects started to produce the sequence. The vocal responses of the subjects were recorded by hand and by tape recorder to permit subsequent analysis.

\section{RESULTS}

\section{Calculation of Repetition Avoidance}

Repetition avoidance $(\phi)$ was calculated as described by Wagenaar (1970), and can be summarized as follows. Expected frequencies of engrams $\left(e_{1} \ldots e_{n}\right)$ are estimated on the assumption of independence of observed frequencies by the formula

$$
f\left(e_{1} e_{2} \ldots e_{n-1} e_{n}\right)=\frac{f\left(e_{1} e_{2} \ldots e_{n-1}\right) f\left(e_{2} \ldots e_{n-1} e_{n}\right)}{f\left(e_{2} \ldots e_{n-1}\right)}
$$

Observed frequencies of repeated engrams $\left(e_{1}=e_{n}\right)$ and nonrepetitive engrams $\left(e_{1} \neq e_{n}\right)$ are then added up to two separate sums. The same is done for expected fre- quencies. Then, the observed frequencies $\left(\mathrm{O}_{\mathrm{i}}\right)$ of repetitions and nonrepetitions are compared with their predicted frequencies $\left(\mathrm{E}_{\mathrm{i}}\right)$ by

$$
\chi^{2}=\sum_{i} \frac{\left(0_{i}-E_{i}\right)^{2}}{E_{i}} .
$$

The coefficient of nonrandomness is given by $\sqrt{\chi^{2} / N}$, in which $\mathrm{N}$ is the number of responses in the series $(120)$. This is normalized for different numbers of alternatives by the approximation formula (Wagenaar, 1972)

$$
\phi=\sqrt{\chi^{2} / \mathrm{N}}(57.27 \mathrm{e} .072 \mathrm{~A}-56.27)+.61 \mathrm{e}^{-.522 \mathrm{~A}+.06 .}
$$

In this way, the extent to which a response $R(i)$ is a repetition of $R(i-1), R(i-2), R(i-3), R(i-4), R(i-5)$, and $R(i-6)$ is measured independently for these six orders. Negative values indicate fewer repetitions and positive values more repetitions than would be expected by chance.

\section{Semantic Similarity}

Repetition avoidance is given in Figure 1. The analysis of variance showed that there was no statistically significant effect of conditions $(F<1)$. The effect of order was significant $[F(5,140)=40.35, p<.001]$. The order $\mathrm{x}$ conditions interaction was not $(\mathrm{F}<1)$.

\section{Acoustic Similarity}

Repetition avoidance in the acoustic conditions is given in Figure 1. Since there are no a priori reasons for differences between alliteration and rhyme, these con. ditions are taken together for a comparison with the acoustically different words. After averaging the scores over order of dependency, the a priori contrast $(-.5$, $-.5,1)$ among the conditions was tested. The $t$ test showed that this contrast was significant $[\mathrm{t}(20)=2.40$,
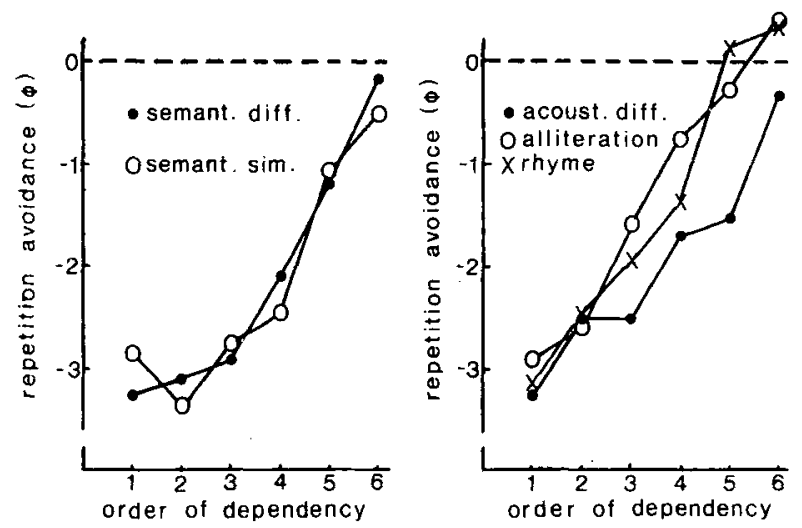

Figure 1. Repetition avoidance in the semantic (left) and acoustic (right) conditions for six orders of dependency. 
$p<.025]$. The analysis of variance showed that order of dependency was significant $[F(5,100)=29.76, p<.001]$. The interaction effect of order $x$ conditions was not sig. nificant $(\mathrm{F}<1)$.

\section{DISCUSSION}

The results suggest that acoustic, rather than semantic, similarity affects repetition avoidance in sequential vocal-response production. For the explanation of this finding, it is necessary to realize that the processes involved in sequential response production may be rather different from those in typical memory tasks, such as the free-recall task. The processes required for repetition avoidance may be described as follows (Wiegersm?, 1982a). First, retention of six or seven recently produced responses is required. At the same time, the set of admissible responses has to be continuously available in order to enable the subject to compare the recently produced responses with the admissible ones and select one that has not occurred recently. This comparison process is assumed to be mediated by a memory search through the recently produced responses in short-term memory. Finally, a decision process is required to determine whether a match is made or not.

Such an analysis of the repetition-avoidance mechanism in sequential response production has striking similarities to the analysis of performance in one particular task used for the investigation of memory span, that is, the missing-item scan task. In that task, the subject is told that, of a set of items (e.g., the numbers 1-13), 12 will be presented in random order and that the missing item has to be reported. Yntema \& Trask (1963) described this as a task in which the person has to make a search through the first list and somehow test items to determine whether they appear in the second. The missingitem task was considered to be one in which searching and testing are most likely the intervening processes between memory and overt response. This analysis is found again in empirical studies with the missing-item task. It has been pointed out that retrieval of the items scanned is not necessary in order to find the missing item (Buschke 1963). This was supported by Madsen \& Drucker (1966), who found that the ability of subjects to find the missing item is a function of storage, rather than of a retrieval process. The hypothesis that two memory sets, representing the actual order of previous responses and their serial order as memorized in advance, can both determine performance has been supported in a number of studies (Buschke, 1967, 1968; Buschke \& Lenon, 1969). All such studies supported the original analysis of the missing-item task by Yntema \& Trask (1963).

The functional similarity of finding a response not recently produced in sequential response production and the missing-item scan in presented material is considerable. This is all the more the case when the missing scan is studied in a continuous presentation task (running missing scan), as was used by Hinrichs and Buschke (1970). The only difference, then, seems to be that, in the task used in this study, the memory set of recent material is produced rather than presented. This might have consequences for the type of memory representation, but leaves the functional equivalence of both tasks intact. From our study, it appears that repetition avoidance in production is mediated at a phonetic level of representation. It seems likely that the memory representation of both response sets (admissible and recently produced responses), as well as their comparison, occurs at a phonetic level of processing.

The absence of semantic-similarity effects suggests that the meanings of words are less important in repetition avoidance than are their sounds. The acousticsinilarity effect found with words also suggests that semantic differences among the responses are not very helpful even when acoustic coding fails. There may be two explanations for these findings. The first is that previous responses are not at all semantically encoded because of the rather high speed of production. It was suggested by Baddeley (1976, p. 120), among others, that encoding for long-term retention needs more time than for short-term retention. A different explanation is that, even when semantic coding occurs, it is not used in repetition avoidance, since only phonetic representations are available to the repetition-avoidance mechanism. Such an explanation is in line with hierarchical models of speech production in which meaning levels are clearly distinguished from form levels of production (Butterworth, 1980). These issues need further research with production tasks.

\section{REFERENCES}

BAdDeley, A. D. (1976). The psychology of memory. New York: Harper \& Row.

Buschкe, H. (1963). Relative retention in immediate memory determined by the missing scan method. Nature, 200, 11291130.

Buschke, H. (1967). Two kinds of short-term storage. Psychonomic Science, 8, 419-420.

Buschke, H. (1968). Perceiving and encoding two kinds of iteminformation. Perception \& Psychophysics, 3, 331-336.

Buschke, H., \& LENON, R. (1969). Ordinal sequence in shortterm retention of numbers. Journal of Experimental Psychology, 81, 201-203.

BUTTE RWORTH, B. (1980). Some constraints on models of language production. In B. Butterworth (Ed.), Language production: Vol. l. Speech and talk. London: Academic Press.

Hinrichs, J. V., \& Buschke, H. (1970). Running missing scan: Perception of oldest member in serial presentations. Psychonomic Science, 19, 125-126.

KeELe, S. W. (1969). Repetition effect: A memory-dependent process. Journal of Experimental Psychology, 80, 243-248.

Mad8En, M. C., \& Drucker, J. M. (1966). Immediate memory by missing scan and modified digit span. Psychonomic Science, 6. 283-284.

Solomon, R. L. (1949). A note on the alternation of guesses. Journal of Experimental Psychology, 39, 322-326.

Sumbr, W. H. (1963). Word frequency and serial position effects. Journal of Verbal Learning and Verbal Behavior, 1, 443-450. 
Uit den BoogaArT, P. C. (1975). Woordfrequenties. Utrecht: Oosthoek, Scheltema \& Holkema.

WagenaAr, W. A. (1970). Subjective randomness and the capacity to generate information. Acta Psychologica, 33, 233-242.

WAOENaA, W. A. (1972). Sequential response bias. Unpublished doctoral dissertation, Institute for Perception TNO, Soesterberg. The Netherlands.

Whaner, M., \& Baird, J. C. (1981). A quantitative analysis of sequential effects with numeric stimuli. Perception \& Psychophysics, 29, 359-364.

Wiegersua, S. (1982a). A control theory of sequential response production. Psychological Research, 4, 175-188.

Wiegergma, S. (1982b). Sequential response bias in randomized response sequences: A computer simulation. Acta Psychologica, 52, 249-256.
YNTEMA, D. B., \& Trask, F. P. (1963). Recall as a search process. Journal of Verbal Learning and Verbal Behavior, 2, 65-74.

\section{NOTES}

1. In Dutch, the initial vowel of all these letters is pronounced as $/ \mathrm{e} /$ in $/$ step/.

2. In the Dutch pronunciation of all these letters, the consonant is followed by /ei/ as in /day/. The initial consonants of $G$ and $J$ are pronounced differently in Dutch: $G$ is a velar plosive, $J$ a palatal continuant. Both are voiced.

(Manuscript received June 20, 1983;

revision accepted for publication September 30, 1983.) 